\title{
Des pratiques de soutien par les pairs dans un Groupe d'Entraide Mutuelle
}

\section{Peer support practices in a Mutual Assistance Group}

\author{
Aziliz Le Callonnec ${ }^{1}$, Véronique Traverso², Emilie Jouin² ${ }^{2}$, et Nicolas Chambon ${ }^{3}$ \\ ${ }^{1}$ Orspere Samdarra, France \\ 2 Laboratoire ICAR UMR 5191 (CNRS, ENS Lyon, Université Lyon 2) et LabEx ASLAN, France \\ ${ }^{3}$ Orspere Samdarra, Centre Max Weber, U. Lumière Lyon 2, France
}

\begin{abstract}
Résumé. En croisant l'analyse d'entretiens à celles de données vidéo enregistrées dans le Groupe d'Entraide Mutuelle (GEM) Envol et Cie à Villeurbanne, l'article identifie et décrit quelques configurations in situ qui permettent de problématiser la mise en place de relations entre pairs, autour des notions de "similarité », "symétrie », "parité ». Ces mises en place sont examinées à partir des positionnements vis-à-vis des pairs aussi bien que des non-pairs (cas d'une réunion avec une animatrice non paire).
\end{abstract}

\begin{abstract}
By combining the analysis of the interviews with those of video data recorded in a Mutual Assistance Group (Groupe d'Entraide Mutuelle, GEM) Envol et Cie in Villeurbanne, the article identifies and describes some configurations in situ that make it possible to problematize the building of peer relations, around the notions of "similarity", "symmetry", "parity". The establishment of these relations are examined on the basis of how the participants position themselves vis-à-vis peers as well as non-peers (case of a meeting with a non peer facilitator).
\end{abstract}

\section{Introduction}

Depuis quelques décennies, la participation est devenue le véritable mot d'ordre de nos sociétés démocratiques. Cet « impératif participatif» (Blondiaux, 2008) touche l'ensemble de la société civile. Les secteurs du sanitaire, du social et du médico-social n'échappent pas à cette nouvelle norme, comme en attestent les lois du 2 janvier 2002 et du 4 mars 2002, favorisant toutes deux l'expression et la participation des usagers de ces secteurs. Depuis 20 ans, les évolutions législatives, mais aussi le soutien de la puissance publique à des dispositifs dits « innovants» ou à des collectifs d'auto-support ${ }^{1}$, ont renforcé cette perspective. L'objectif est clair : remettre la personne au centre, qu'elle ait un rôle déterminant sur toutes les décisions qui la concernent, afin qu'elle soit actrice à part entière de sa propre vie et de celle des autres, un « usagent» (Heijboer, 2019), agissant « en tant que sujet de son devenir et non en tant qu'objet d'aide» (Ebersold, 2002: 285). Mettre en œuvre ce principe de participation, ce n'est donc pas seulement permettre à la personne concernée de «prendre part » (Zask, 2011) aux décisions et réunions la concernant, c'est aussi lui donner les outils

${ }^{1}$ Ces collectifs d'auto-support, selon la typologie crée par Aurélien Troisoeufs et Benoît Eyraud, sont des organisations centrées sur l'échange et le partage régulier d'expériences entre pairs (soutenu principalement par la parole). Ces collectifs s'organisent autour de l'aide mutuelle. Les groupes des alcooliques anonymes ou des narcotiques anonymes en sont de bons exemples (Troisoeufs et Eyraud, 2015). 
pour devenir véritablement actrice en redistribuant le pouvoir, ce qui implique de favoriser son autonomie, son pouvoir d'agir, son empowerment, mais aussi de reconnaître ses compétences, ses atouts, ses « capabilités » (Sen, 2012), voire ses savoirs expérientiels. La personne dispose de ressources internes et est dotée d'un "potentiel caché » (Ehrenberg, 2020) qu'il s'agit de faire surgir de multiples façons. Cette expérience singulière lui fait acquérir un certain nombre de connaissances et de compétences propres qui lui permettent d'aller mieux et, qui sont autant d'atouts l'autorisant par la suite à venir en aide à ses pairs.

Ce «nouvel esprit du soin» (Ehrenberg, 2021) favorise la prise en compte des acquis expérientiels et incite également le politique à développer et à promouvoir progressivement l'idée déjà ancienne de self-help, de soutien et d'accompagnement par les pairs, soit tout un ensemble de pratiques et de formes d'actions jusqu'alors auto-développées (Gardien et Laval, 2018), qui favorise la participation des " pairs » dans une relation de pair(s) à pair(s) fondée sur le partage d'une expérience commune (de la vulnérabilité, du trouble psy, du handicap, du cancer, du SIDA, des violences sexuelles et sexistes, des addictions, de l'accompagnement d'un proche, etc.), que l'on nomme et que l'on regroupe souvent aujourd'hui sous le terme de " pair-aidance », alors qu'une myriade de pratiques et de termes existent, chacun renvoyant à des manières spécifiques de faire, qui peuvent être informelles et/ou professionnelles.

Ce sont ces pratiques de soutien entre pairs - de plus en plus reconnues du fait qu'elles apparaissent comme d'une grande aide aux personnes qui en bénéficient (Chambon et al., 2020) - et cette figure du pair, qui nous intéressent dans cet article. "Que fait le pair ? ", "Comment faire le pair?" sont les questions qui nous animent. Il s'agit de comprendre comment le pair se positionne comme « pair» dans ses interventions (1) vis-à-vis des pairs et (2) vis-à-vis des non-pairs, en analysant en détail les spécificités des pratiques interactionnelles qui, à la fois, montrent et construisent la relation. Ce n'est donc pas tant ce que les personnes disent d'être pair, de faire le pair, de se former comme pair, d'être accepté ou non comme pair qui nous occupe au premier chef, mais plutôt de comprendre ce qui peut faire d'une rencontre, une rencontre entre pairs.

\section{Différents pairs}

Il nous semble important, avant de nous intéresser à l'interaction en elle-même et à son analyse, de donner des repères sur cette notion de « pair ». D'autant que la compréhension et la conceptualisation d'être/de faire le pair sont loin d'être unifiées ${ }^{2}$, les pratiques elles-mêmes étant très différentes les unes des autres. On peut être/faire le pair d'une multitude de façons aujourd'hui : on peut être pairémulateur, pair-accompagnateur, travailleur pair, facilitateur pair, médiateur de santé pair, pair-aidant, pair-formateur, pair-praticien... et faire du peer tutor, du peer mentoring, du pairing, du self-help, du peer support ou encore du peer counselling.

Le Centre National de Ressources Textuelles et Lexicales ${ }^{3}$ définit le pair comme une "personne de même situation sociale, de même titre, de même fonction qu'une autre personne ». La définition renvoie donc à l'idée de mêmeté. Pour autant, cette motivation lexicale ne nous éclaire pas pour comprendre le soutien entre pairs. Ce n'est pas le fait d'être semblable qui fait d'une personne un pair mais le fait de la reconnaître comme tel, de s'identifier à elle. C'est là un premier point définitionnel que nous retenons : il faut une

\footnotetext{
${ }^{2}$ Il suffit pour s'en convaincre de se reporter aux différents points de vue exprimés dans Rhizome 7576 , à travers des entretiens avec des pairs en santé et des écrits de chercheurs. L'accent est souvent mis sur des aspects différents des interventions des pairs.

${ }^{3}$ https://www.cnrtl.fr/definition/pair
} 
reconnaissance mutuelle, une identification réciproque, un sentiment d'appartenance à une même communauté d'expérience (Gardien, 2018).

\section{1 Être reconnu comme pair}

Les pairs ne sont pas identiques en tout point et ne sont pas à proprement parler exactement les mêmes. Ils peuvent avoir des conditions de vie très différentes les uns des autres, des appartenances sociales très dissemblables, venir de milieux sociaux aux antipodes, avoir des rôles sociaux et une multitude d'identités variées et parfois contraires, ils peuvent même avoir vécu une même expérience mais de manière très différente. Ils ont chacun leur individualité, leurs identités et expériences propres. C'est ce que l'on peut rapidement constater en allant dans un groupe d'entraide mutuelle (GEM) par exemple. Les adhérents viennent et participent à la vie d'un tel collectif parce qu'ils se reconnaissent comme ayant vécu une expérience de la souffrance psychique et/ou de l'isolement social mais, au-delà de ce point commun, ils ont des vies et des parcours très hétéroclites : on peut y retrouver des retraités, des personnes âgées, des étudiants, des personnes en situation de handicap, sans domicile fixe, vivant ou ayant vécu avec des troubles psychiques ou non, vivant ou ayant vécu une ou des périodes d'hospitalisation ou non, etc.

Considérer l'autre comme un pair ne va donc pas toujours de soi (pour les adhérents du GEM enquêté par exemple, parler de «pair-aidance » n'est pas évident) ${ }^{4}$. La parité n'est pas un fait naturel et, c'est d'ailleurs ce que met en exergue Dominique Launat. Voici ce qu'il nous en dit : " on définit souvent ces notions [de "pair" et de "pair-aidance"] comme des états... de fait. Ce ne sont, selon moi, que des attributions, des désignations, des imputations... On les détermine comme des faits avérés et il irait de soi que tout un chacun se reconnaisse dans ce "semblable" qu'on (lui) désigne ou attribue. Et l'on tient pour certain que le pair (aidant) va jouer un rôle facilitateur dans la prise en charge et/ou l'accompagnement du pair (aidé ?) » (Launat, 2020 : 42). Mais, « il n'y a de pairs que parmi celles ou ceux que je reconnais comme tels. Cela peut être convenu ou conçu par un tiers (la société ?) mais il me revient de me conformer ou non à la parité à laquelle on me convie » (ibid. : 43).

Être pair passe donc par la reconnaissance du « dénominateur commun qui fait de l'autre un pair» (Niard et al., $2020: 22$ ). Le cas d'Antoine, qui souffre de troubles psychiques, et consomme du cannabis, rapporté par Niard et al., illustre très bien cela. A l'hôpital de jour, il est mis en lien avec un médiateur de santé pair ${ }^{5}$, lui-même pris dans une problématique addictive à la nicotine et au cannabis. Celui-ci témoigne de la mise en place de leur relation, et souligne l'importance de sa propre expérience et de ses savoirs d'usage. Ils sont détenteurs d'une même " culture déviante », ayant tous deux fait l'apprentissage de l'usage du cannabis, des techniques propres à cet usage (il faut apprendre à fumer " comme il faut " pour pouvoir «planer»), mais aussi de ses effets (il faut être capable de prendre conscience que l'on « plane », il faut donc apprendre à en reconnaître les symptômes), qu'il convient d'apprendre à apprécier progressivement (il faut redéfinir ces effets et les considérer comme agréables) pour finalement " concevoir la drogue comme source de plaisir» (Becker, 1985). Ils sont tous deux devenus fumeurs, sont passés par les mêmes étapes et sont donc porteurs d'un vécu expérientiel commun, qui transparaît dans la relation. En effet, Antoine « a rapidement pu se rendre compte de mes connaissances pratiques et intimes sur le sujet. Nous partagions un langage commun spécifique ; une espèce de jargon propre aux consommateurs de cannabis.

${ }^{4}$ Comme le formule Launat, « on ne désigne pas, dans un GEM, du pair à priori » (2020:44).

${ }^{5}$ Les médiateurs de santé pairs désignent une forme d'entraide professionnalisée entre pairs. Ce sont de nouveaux professionnels, intégrés dans les équipes de soins et rémunérés. Il s'agit de l'une des premières formes de professionnalisation d'usagers en France (Troisoeufs, 2020). 
Nous parlions de la même chose et de la même manière. C'est plus par l'expression orale que par le récit d'expériences qu'un processus d'identification a débuté » (Niard et al., $2020: 19$ ). C'est tout un ensemble de petits indices, de signes semés au cours des échanges (verbaux ou non-verbaux) qui permet ce processus d'identification.

\subsection{Partager une expérience commune}

L'exemple précédent introduit parfaitement notre deuxième apport définitionnel : pour être pairs, il faut partager une même expérience ou, plus spécifiquement, les pratiques de soutien entre pairs « se fondent sur le partage des expériences vécues » (Laval et Gardien, 2020). Ils ont vécu ou pensent avoir vécu une expérience similaire et, du fait qu'ils ont cela en commun et qu'ils le reconnaissent, ils s'entraident et se soutiennent. Cette même expérience crée une certaine proximité qui favorise et facilite les relations. Être pair, c'est être dans un rapport de similarité, de symétrie, d'horizontalité, d'égalité. Ces rencontres se distingueraient donc de celles qu'ils peuvent avoir avec ceux n'ayant pas cette proximité expérientielle, le pair comprenant mieux la situation l'ayant lui-même vécue. Avec ses pairs, la face qu'il faut garder ou la «bonne figure» (Goffman, 1974), si on les comprend comme des lignes de conduites (Joseph, 1998), sont moins contraignantes que dans beaucoup d'autres rencontres sociales.

C'est le cas par exemple des participants aux groupes de soutien organisé par le réseau français sur l'entente de voix (REV). Tous les participants entendent des voix. Cette expérience est partagée par tous, ce qui la normalise et la banalise, ce qui permet également de mieux la comprendre, de lui donner du sens. Les participants peuvent parler plus sereinement de comment ils vivent ces "phénomènes» au quotidien. Ils savent qu'ils peuvent en parler «sans tabou », "dans un climat de confiance et de sécurité partagées » (Golf et al., 2020), parce qu'ils sont entourés de pairs qui ne remettent pas en cause la véracité de leur expérience. Ils peuvent donc les comparer et y trouver, progressivement, un sens commun, une façon propre de les comprendre et de s'y adapter. Ils créent ainsi tout un ensemble de savoirs pairjectifs, valables pour tous les pairs. La pairjectivité est ainsi une disposition du pair qui établit de nouvelles expériences et savoirs en les fondant sur son expérience propre, et en la confrontant à celles de ses pairs à travers une "perspective cognitive collective » (Gardien, 2020).

L'accent est mis sur ce qui les rassemble et les unit : cette expérience commune. En pratique, dans les rencontres, faire le pair se traduirait donc par mettre en avant ce qui nous rapproche (et non ce qui nous distingue), par se positionner en symétrie (et non en asymétrie), au niveau de l'expérience. Faire le pair implique de créer du semblable: c'est une action de rapprochement.

Mais, créer du semblable, c'est aussi et toujours créer de la différence. Comme l'explique Gardien (2018), « l'expérience est instituée comme marqueur de distinction sociale. Elle est un critère de séparation entre "eux" et "nous" ». La parité prend donc forme dans un cadre dual. Être pair se pose nécessairement par rapport à un cadre où il y a aussi des non pairs (sinon en tant qu'êtres humains, nous serions tous des pairs). Tenter de définir ce que signifie faire le pair, c'est s'intéresser aux rapports qui lient les individus « les uns avec les autres », « les uns pour les autres », mais aussi « les uns contre les autres» (Simon, 1991). Faire le pair, c'est donc se poser vis-à-vis de ceux que l'on identifie comme des pairs, mais aussi visà-vis des non-pairs, de ceux que l'on maintient à distance.

C'est cette dualité entre parité, proximité, similarité, symétrie et différence, distance, asymétrie que nous allons retrouver dans l'analyse de corpus qui suit. On y observe, d'une part, comment se met en place un collectif sur la base de l'affirmation du partage d'une 
expérience et, d'autre part, comment de la différence est ré-insufflée, par petites touches, dans la parité activement construite par l'ensemble des participants.

\section{Méthodologie et contexte de l'étude}

Nous abordons ces questions à partir d'un de nos terrains pour le projet de recherche TIASAMN $^{6}$, à travers l'étude des pratiques, c'est-à-dire en observant et décrivant très précisément ce que font les personnes aux cours de leurs rencontres. Pour aborder les choses sous cet angle, nous nous basons sur l'analyse d'enregistrements vidéo in situ, que nous mettons en écho avec des entretiens. Ces enregistrements nous permettent d'analyser le détail des pratiques, et de mettre l'accent sur des actions ou activités descriptibles.

\subsection{Le GEM}

Créés suite à la promulgation de la loi du 11 février 2005, les GEM sont des structures associatives visant à la fois la "réinsertion » (sociale, professionnelle et citoyenne), le développement du lien social ainsi qu'une meilleure participation sociale des personnes présentant des troubles similaires, via l'émergence d'un espace neutre leur permettant de discuter, de se soutenir, de s'entraider mutuellement. Ce sont des structures atypiques : elles ne sont associées ni au secteur sanitaire, ni au secteur médico-social. Il ne s'agit pas de structures spécialisées dans l'accompagnement ou la prise en charge du handicap psychique, même si elles sont construites et pensées comme des outils de prévention et de compensation de celui-ci. Les GEM incarnent « une nouvelle façon de faire et de nommer le travail thérapeutique », sans « la dimension thérapeutique des lieux de soins » (Troisoeufs, 2012). Ils proposent des espaces construits «par » et " pour » leurs adhérents, qui valorisent leurs expériences et leurs connaissances, expériences qui sont mises à profit d'autrui en suivant un principe de soutien mutuel.

La captation vidéo s'est déroulée au sein du GEM Envol et Cie, association qui est auto-gérée par les adhérents ${ }^{7}$. Elle est ouverte tous les après-midis aux adhérents, du lundi au vendredi, de $14 \mathrm{~h}$ à $17 \mathrm{~h}$ pour discuter, partager un moment convivial dans le salon, participer à un temps d'activité, etc. Nous nous intéressons dans ce qui suit à l'enregistrement d'une de ces activités : l'atelier radio ${ }^{8}$.

${ }^{6}$ TIASAMN « Tiers-intervenants dans l'accès à la santé et au numérique » est un projet Pack Ambition Recherche financé par la Région Auvergne-Rhône-Alpes de 2019 à 2024, et porté par le laboratoire ICAR en partenariat avec l'ORSPERE-SAMDARRA.

${ }^{7}$ L'Ancreai (2017) s'interroge sur les modes de gouvernance des GEM. Il distingue trois modes de gouvernance au sein de ces structures. Un premier ensemble de GEM sont encore fortement dirigés « de l'extérieur». Un second ensemble comprend des formes intermédiaires. Enfin, un dernier ensemble comprend les GEM au sein desquels les usagers réfléchissent (à) et conçoivent leurs propres manières de gouverner leur structure. Ils sont autogérés.

${ }^{8}$ Cet atelier radio est assez singulier. Contrairement aux autres ateliers hebdomadaires proposés par le GEM (écriture, piscine, relaxation, broderie...), il n'est pas animé par un adhérent (appelé alors « initiateur »), mais par l'une des animatrices du GEM. Il est important de le mentionner, car les activités/ateliers sont censés être l'apanage des adhérents, et l'équipe d'animation intervient généralement très peu lors des autres ateliers : ils sont uniquement là « en cas de besoin » et interviennent « le plus discrètement possible». 


\subsection{L'extrait choisi}

L'extrait provient de la deuxième réunion de l'année de l'atelier radio. Elle vise à préparer des interventions dans une radio locale, Radio Canut. Y participent une des animatrices et huit adhérents. L'extrait se situe au tout début de la réunion, au moment où l'animatrice propose de commencer par un tour de table de présentation de chacun.

Au départ, il y a au moins trois statuts différents parmi les participants :

- l'animatrice, qui est une professionnelle salariée du GEM ;

- les personnes adhérentes intéressées à s'investir dans l'atelier ;

- l'enquêtrice (qui est aussi adhérente, mais qui ne participe pas à l'activité), que l'on voit passer de loin en loin pour s'occuper des caméras.

Le tour de table de présentation est intéressant pour une investigation de la problématique des pairs. C'est en effet un format qui conduit chacun à prendre la parole à son tour, pour exposer de soi une identité en cherchant à la rendre appropriée au contexte (Greco, 2006). Les prises de paroles successives se construisent à la fois par rapport :

- à la tonalité du tour de table initié par l'animatrice, et aux consignes qu'elle donne au départ ;

- $\quad$ aux prises de parole précédentes dans le tour de table. Sur ce point, Greco, dans le contexte qu'il étudie, parle de socialisation aux fonctionnements de l'activité "présentation de soi", pour désigner le fait que, peu à peu, les participants apprennent à calibrer leurs interventions en longueur, à ne pas en faire trop et à savoir choisir les éléments les plus pertinents (2006:158);

- au contexte (le tour de table, l'atelier radio, le GEM), auquel les participants s'adaptent tout en le configurant.

Sur le plan séquentiel et organisationnel, un tour de table de présentation s'effectue généralement en suivant la disposition spatiale des participants (passant d'une personne à sa voisine autour de la table). Les études existantes ont aussi montré que le locuteur dont c'est le tour donne des indices verbaux et corporels de cession imminente de son tour (changement $\mathrm{du}$ ton de voix et de la prosodie, changement de la position du corps : la personne s'avance lorsqu'elle commence ou s'apprête à parler et recule lorsqu'elle finit). Bien souvent, par un petit signe (gestuel, visuel ou postural), elle indique aussi à la personne voisine qu'elle peut démarrer (Greco, 2006 ; Traverso, 2012). Les descriptions ont aussi mis en évidence que l'ordre de déroulement du tour de table peut être signifiant, par exemple lorsqu'un participant prend la parole en dérogeant à l'organisation spatiale attendue (Traverso, 2021).

Dans le cas qui nous occupe, on pourrait considérer a priori que l'initiation du tour de table par l'animatrice, et ce qu'elle demande qu'on y dise, propose des positionnements aux personnes présentes, qui se trouvent de fait mises, sinon dans un statut de pair, tout au moins en position symétrique les unes par rapport aux autres, alors que l'animatrice, elle, se place dans une position asymétrique. Il est donc intéressant d'observer comment ces positionnements a priori sont ensuite incarnés, construits ou déconstruits dans les pratiques des adhérents.

Au niveau du contenu, la présentation de soi dans un tour de table pourrait favoriser l'essai de s'individualiser. Mais on observe, d'une part, que reprendre la tonalité, le format, etc. des présentations successives est une importante ressource utilisée par les personnes, et qu'elle peut conduire à gommer les spécificités. Comme le dit encore Greco : « Combien de fois chacun de nous s'est retrouvé dans l'angoisse de choisir les bons dispositifs identitaires adéquats au contexte ? Combien de fois, on s'est senti soulagé car notre tour venait assez tard et du coup on pouvait "bricoler" une présentation de soi à partir de celles qui nous avaient précédées? » (2006:157). 
D'autre part, on peut aussi faire l'hypothèse que le tour de table de présentation est une pratique qui permet de « faire groupe », de mettre donc en avant ce qui est commun, ce qui nous réunit, ce que l'on partage. Peut-on associer cette dimension de «faire groupe » à faire les pairs?

\section{Analyses}

\subsection{Le lancement du tour de table}

L'animatrice initie le tour de table en donnant son prénom et sa fonction au sein du GEM. Puis elle présente son expérience à Radio Canut, les motivations qui l'ont poussée à proposer cet atelier radio et son intérêt pour le partage d'information via ce canal. Dans l'extrait suivant ${ }^{9}$, elle met principalement en avant le collectif, par l'emploi d'expressions évoquant le partage, la rencontre («partager des opinions », «j'ai voulu le partager », « un moyen de se rencontrer ») et par la répétition itérative du mot « ensemble » :

\section{Extrait 1 :}

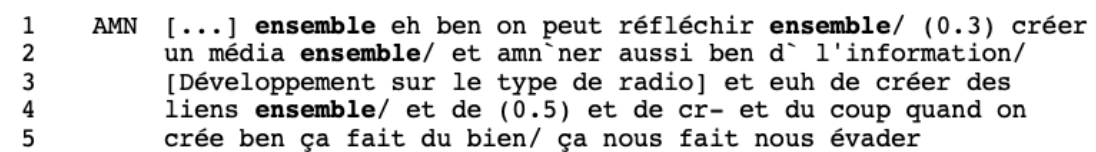

Elle caractérise ainsi ce qui se joue dans l'atelier, à savoir créer une unité, faire ensemble pour créer du lien (comme on le voit dans la fin de l'extrait). Cette accentuation forte sur le collectif illustre le rôle même de l'animatrice. Travailler à partir du groupe, mettre en mouvement un collectif, soutenir les liens existants entre les personnes adhérentes et être garante du cadre dans lequel se déroulent ces interactions est ce que l'on attend d'elle. Son rôle de professionnelle implique de faciliter la fonction de «métier à tisser des liens » du GEM (Fiacre et al., 2017).

La dimension de « bien-être » se retrouve aussi lorsqu'elle évoque le fait de «parler de ce qu'on aime ». À la fin de sa propre présentation, elle invite les participants à poursuivre le tour de table en leur proposant d'axer leur prise de parole sur leur expérience antérieure de l'atelier ou leur intérêt pour la radio :

\section{Extrait 2 :}

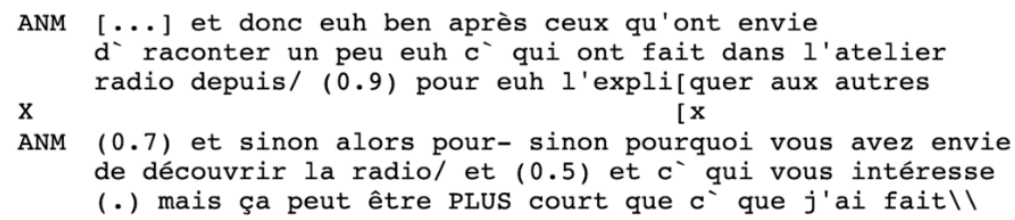

Bien qu'elle souligne qu'il faut faire selon son envie (lignes 1-2), elle propose bel et bien un cadre pour les prises de parole.

9 Les conventions de transcription utilisées sont les conventions ICOR : http://icar.cnrs.fr/projets/corinte/documents/2013 Conv ICOR 250313.pdf. La notation des gestes est consultable sur le site CORVIS http://icar.univ-lyon2.fr/projets/corvis/. 


\subsection{Un tour de table en étoile}

À la fin de son tour, l'animatrice désigne son voisin de droite comme celui qui va enchaîner, en s'orientant clairement vers lui (tête et regard), au moment où elle cesse de parler :

\section{Extrait 2bis :}

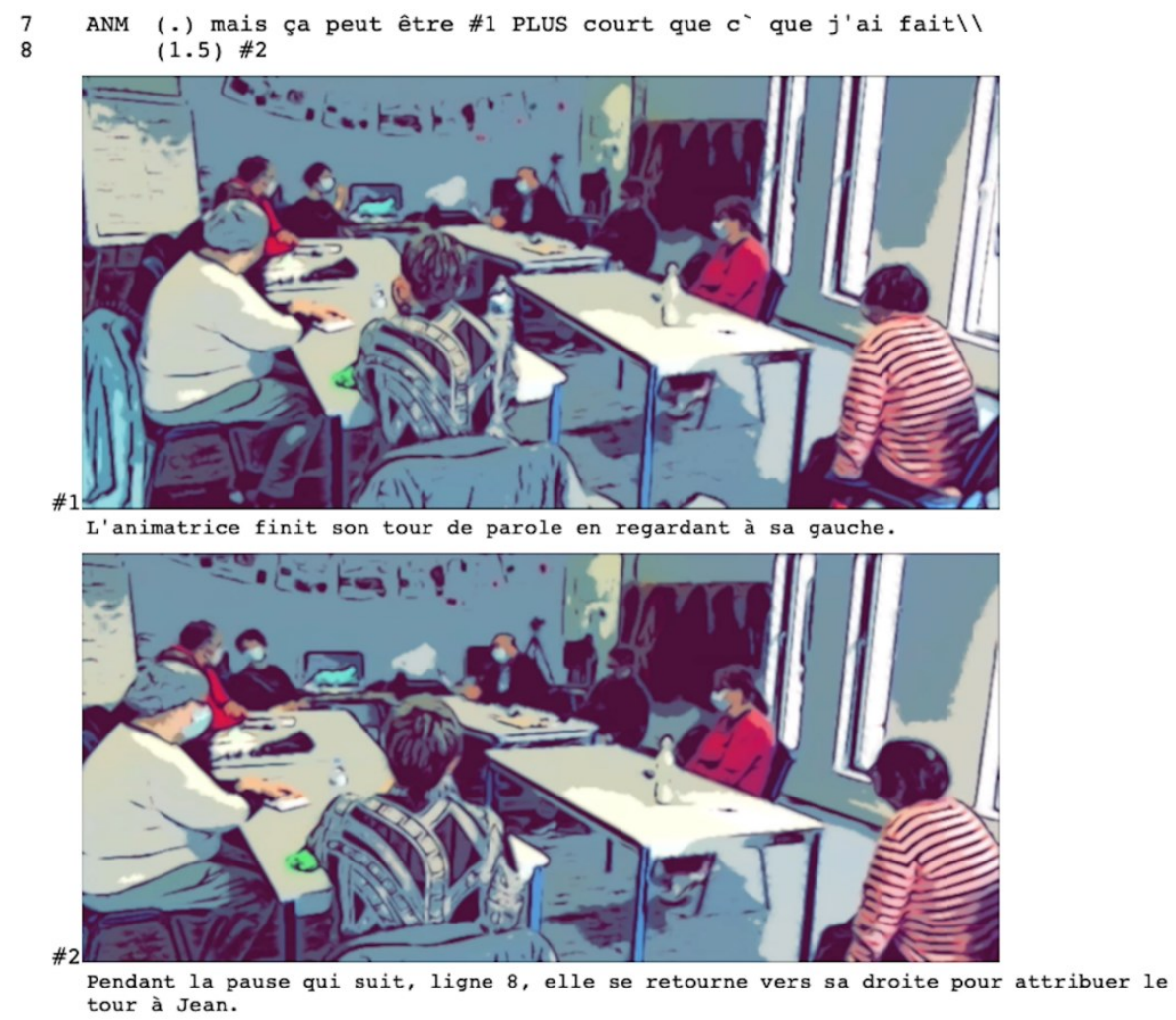

En désignant, même si ce n'est que gestuellement, le premier adhérent à parler, elle met en place un ordre attendu pour le déroulement du tour de table (vers la droite). Pourtant, à la fin du tour de parole de Jean, cet ordre est modifié par Farouk :

\section{Extrait 3 :}

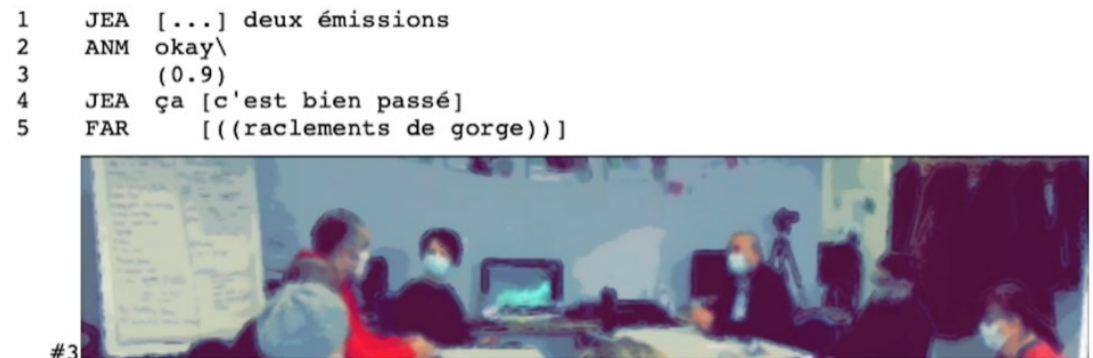

Pendant que JEA finit son tour, ANM le regarde et FAR de l'autre côté de la table produit des petits raclements de gorge.

6

ANM ((petit rire))

((3.6, Farouk fait des petits gestes de la main et se racle la gorge)) 

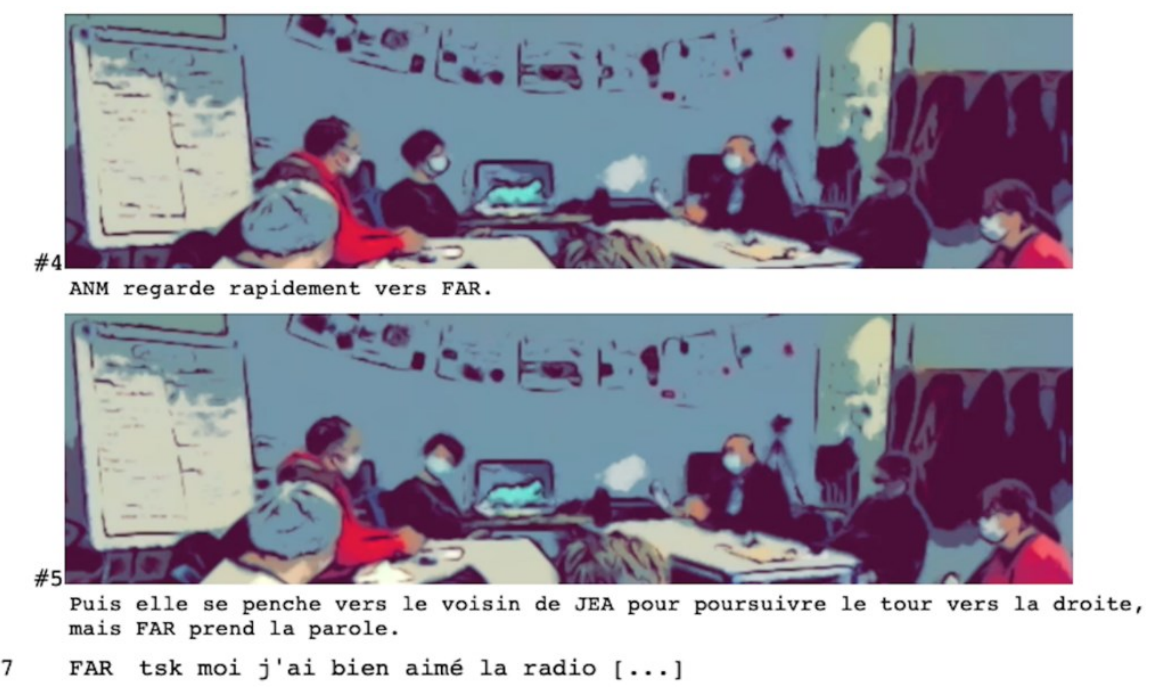

Malgré l'intervention, toujours silencieuse, de l'animatrice pour engager le voisin de Jean à poursuivre, Farouk s'attribue la parole et inverse la direction du tour de table, qui progressera jusqu'à la fin vers la gauche. Ce même participant dérogera encore deux fois au fonctionnement attendu du tour de table, en reprenant la parole entre deux autres adhérents pour ajouter des commentaires, alors que tout le monde n'a pas encore parlé (voir 4.3.3).

Ce qui se passe jusque-là est intéressant à plusieurs titres.

Tout d'abord, cela fait apparaître le rôle continu de l'animatrice dans le fonctionnement de l'activité. Elle a donné le cadre au départ. Elle est celle à qui les adhérents s'adressent lorsqu'ils parlent (ils la regardent), et elle produit des régulateurs (ex. extrait 3, ligne 2), se positionnant ainsi comme la réceptrice principale et l'organisatrice du tour de table. Elle veille aussi aux contenus, n'hésitant pas à « recadrer » les participants, quitte à les interrompre :

\section{Extrait 4 : Début de la prise de parole de Jean}

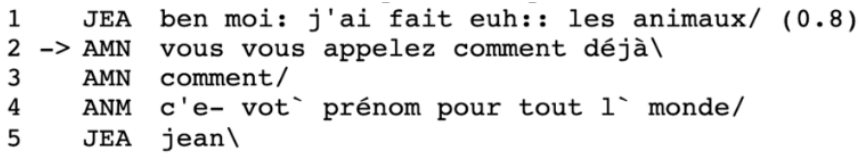

\section{Extrait 5 : Début de la prise de parole de Farouk}

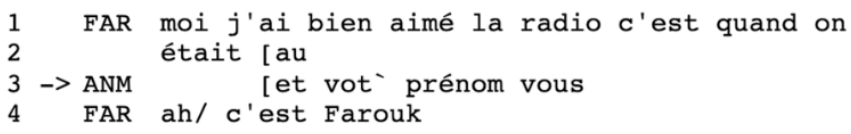

Commencer par dire son prénom et se situer par rapport à Radio Canut deviennent une manière de faire acquise dès le troisième participant.

La mise en place du cadre de l'activité passe aussi par d'autres types d'interventions sur les contenus dont l'animatrice accompagne les prises de parole des adhérents, veillant à leur progression et à leur cohérence. Elle relance parfois une prise de parole pour coller au cadre : 


\section{Extrait 6 : Jean}

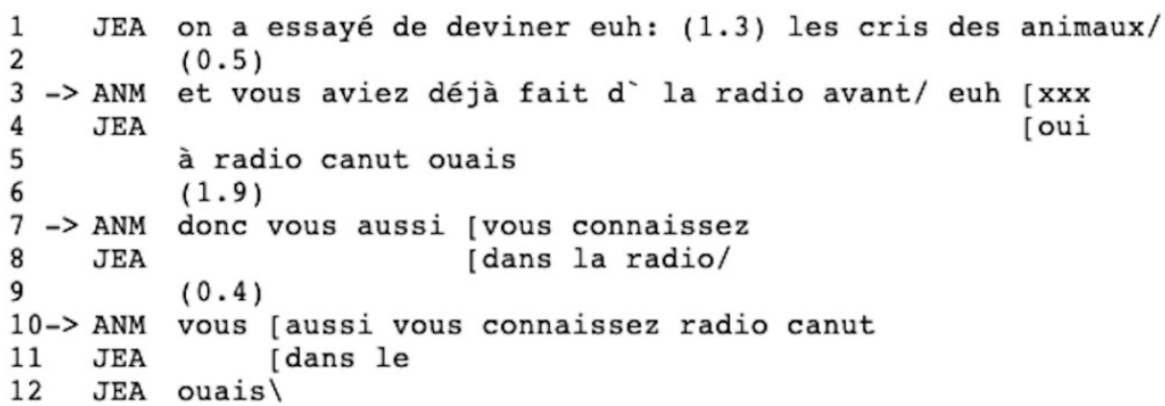

Enfin, lorsque les prises de parole semblent dévier, elle les interrompt en rappelant l'activité en cours ( du coup on termine le tour », « on termine le tour ») ou bien elle demande au participant d'expliquer pourquoi il aborde ce sujet :

\section{Extrait 7 : Quentin est en train de parler de son frère Orel qui a donné des cours de musique au GEM}

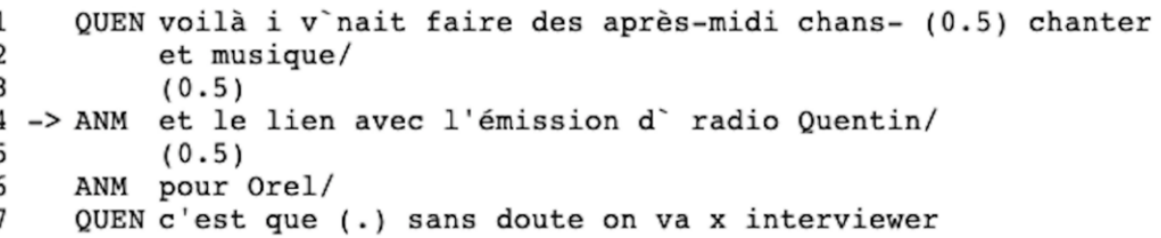

On voit donc que le cadre est solidement tenu, et que les adhérents s'y conforment plutôt volontiers. Par rapport à notre questionnement sur « faire le pair », apparaît ici, d'une part que l'animatrice ne se positionne clairement pas comme paire des adhérents au niveau de son comportement interactionnel. Sur ce plan, son travail vise plutôt à « créer de la parité » entre les personnes, puisqu'elle organise une démonstration de " partage d'expérience » (de la radio). Il est intéressant d'observer qu'au fil du tour de table, ce partage d'expérience est étendu par les adhérents à la (pratique ou l'écoute de la) musique, thème choisi pour la prochaine émission de radio, et plus largement au GEM lui-même. Ainsi faire ou avoir fait de la radio, aimer l'activité radio, être allé à Radio Canut, aimer la musique ou en avoir déjà fait (voire avoir un frère musicien pour un des participants), être un ancien du GEM, avoir fait beaucoup d'activités au GEM, créent véritablement un lieu commun ${ }^{10}$ permettant d'affirmer un partage, dont l'animatrice est, cette fois, partie prenante («moyen de se rencontrer et de créer des liens ensemble »).

Ce début du tour de table montre aussi que, parallèlement à l'affirmation du partage, s'expriment des disparités, des asymétries et des positionnements singuliers. Nous allons en examiner quelques cas, pour réfléchir à la question : parité, similarité, symétrie ou leurs contraires.

\footnotetext{
${ }^{10}$ On ne peut pas ne pas penser aux lieux communs de la conversation, qui permettent de se reconnaître comme membre d'un groupe (Traverso, 1993 ; Plantin, 1993).
} 


\subsection{Se positionner comme pair : entre distance et proximité}

\subsection{1 Être le plus ancien, expérimenté, passionné...}

On est frappé au visionnement du tour de table par la tendance de certains participants à passer de « avoir, et donc partager, l'expérience (de la radio, de la musique ou du Gem) » à avoir la plus ancienne, plus diversifiée, plus précise expérience de ces activités. On peut voir là affleurer des asymétries au sein de ce qui se donne au départ comme une symétrie. Différents types de formulations contribuent à leur mise en place.

\section{Extrait 8 : Début du tour de Quentin}

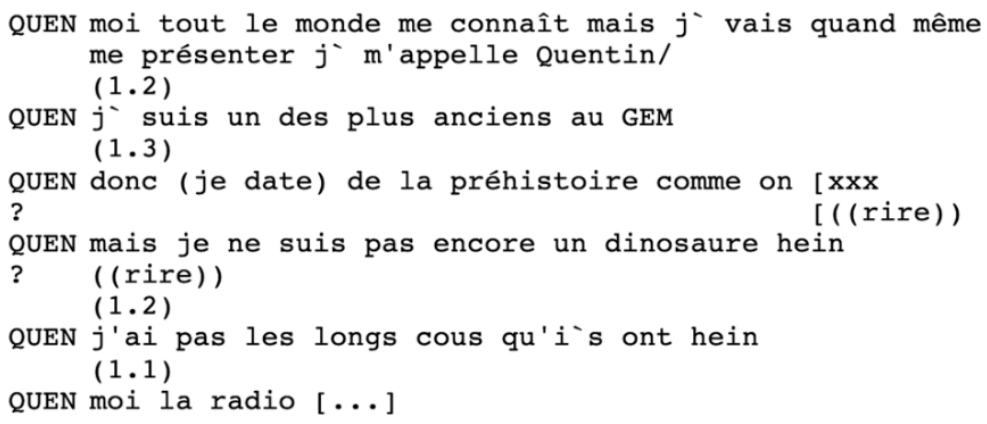

Le préambule de Quentin (avant qu'il en vienne à la radio, ligne 13) le positionne de façon singulière (un des plus anciens, qui n'a pas besoin de se présenter, un dinosaure comme un pater familias). L'utilisation d'une plaisanterie $(6,8,11)$ le distingue aussi des autres participants, toujours sérieux, d'autant qu'il la formule en regardant le groupe, alors que les autres participants (à part Farouk, et dans une moindre mesure Armelle) restent orientés vers l'animatrice pendant qu'ils parlent, ce que Quentin ne fait que lorsqu'il aborde la question de la radio.

Alors que certains participants évoquent leur expérience de la radio par une description succincte et précise de ce qu'ils y ont fait (« deux émissions », «j'avais écrit des textes qu'on a diffusés », «j'ai raconté un peu mon histoire un peu que j'ai vécue en Tunisie jusqu'à l'âge de douze ans »), d'autres recourent à des énumérations et à l'expression du haut degré, qui participent d'une forme de surenchère :

\section{Extrait 9 : Quentin}

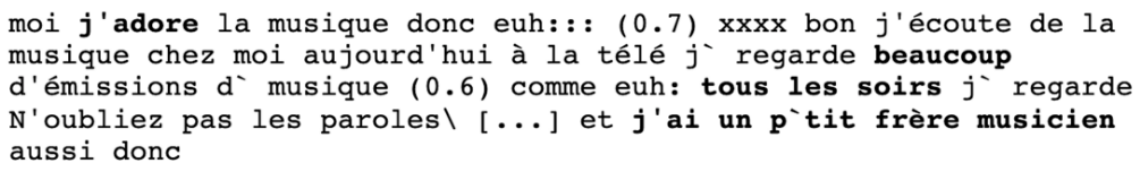

\section{Extrait 10 : Armelle}

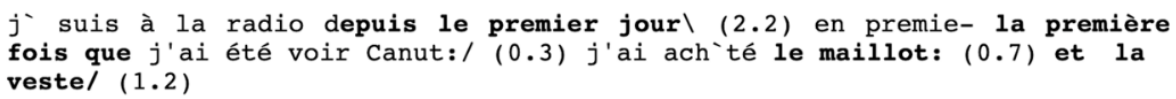

\section{Extrait 11 : Catherine}

la musique c'est pareil/ ça me parle parce que (0.5) j'ai fait du piano $j$ 'ai fait de la flûte: euh $(0.4) j$ 'avais commencé un peu la guitare 
Chacun à leur manière, ces différents adhérents mettent en place une forme d'argumentation pour montrer, voire démontrer à l'aide de leurs constructions rhétoriques, à quel point (et plus que les autres) ils partagent le commun.

Ceci montre bien que le similaire, commun, symétrique repose, dans son détail, sur une organisation de multiples petites différences, spécificités, gradations et asymétries, qui n'entachent pourtant pas le partage manifesté, tant que chacun l'accepte, ne s'en offusque pas ou ne la remet pas en cause.

\subsubsection{Accueillir quelqu'un}

Le collectif que le tour de table contribue à affirmer se manifeste aussi dans la façon d'accueillir une des participantes, nouvellement arrivée :

\section{Extrait 12 : Valentine}

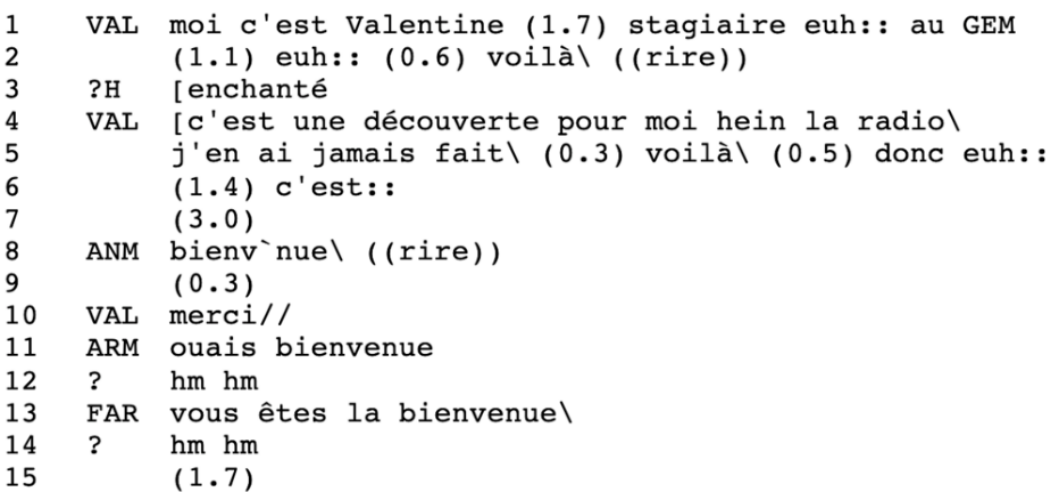

Valentine se conforme tout à fait au cadre proposé pour sa présentation (prénom, statut par rapport au GEM, expérience de la radio). Elle est la première personne du tour qui énonce n'avoir aucune expérience de la radio (lignes 4-6), et son tour de parole est assez laconique (très longues pauses, bribes inachevées « donc euh:: (1.4) c'est:: », répétition du marqueur « voilà »). La façon dont est reçue sa présentation est intéressante dans notre réflexion sur la constitution du collectif. Tout d'abord, à la ligne 3, un des membres masculins du groupe (probablement Farouk) répond comme après une présentation en face à face avec une personne dont on fait la connaissance («enchanté »). On peut voir cette pratique comme attribuant à Valentine un statut de personne qui n'est pas (encore) dans le collectif. On note ensuite qu'un très long silence suit la deuxième partie de son tour de présentation, ligne 7 . On peut comprendre que le tour de la ligne 6, restant en suspens sur un énoncé inachevé, les autres participants attendent une forme de complétion. Comme cette complétion ne vient pas, l'animatrice recourt, ligne 8 , à un autre procédé, relevant de son rôle de garante du déroulement de l'activité : une routine d'accueil « bienvenue ». Par cet usage, elle démontre cet « art de l'ajustement» dont elle est détentrice, en tant que professionnelle du care $^{11}$, qui lui permet d'intervenir subtilement «en toute discrétion » et "juste au bon moment» (Molinier, 2010). Elle sait «être en retrait s'il le faut» mais aussi «être force de propositions » quand c'est nécessaire (Le Callonnec, 2021). Avec cette formule, on pourrait dire qu'elle fait passer Valentine du statut d'« inconnue que l'on vient de rencontrer» ( « enchanté »), à personne que l'on fait entrer chez soi, dans le groupe, dans le collectif. Et il est remarquable que plusieurs autres participants fassent écho à cette formule $(11,13)$,

${ }^{11}$ Le care est selon Molinier, « un geste ou une façon de faire (ou de ne pas faire) ajustés ou accordés aux besoins du destinataire, fussent-ils de distance ou de détachement » (2010:164-165). 
entérinant l'entrée de Valentine dans le collectif de l'atelier radio, et faisant groupe par ce fait même.

\subsubsection{Sortir du cadre}

Les positionnements ancrés dans la différence et la singularité se manifestent aussi par des « entorses » au cadre initialement constitué par l'animatrice. Certains adhérents s'éloignent de l'activité en cours pour aborder des thèmes ou des expériences qui sont propres à leur vécu, à leur envie. Ces digressions sont de nature à conduire vers une individualisation des discours, et elles sont détournées par l'animatrice qui veille, relance l'activité et remobilise l'idée de partage d'envies et d'informations visant à reconstruire le groupe. Elles sont néanmoins intéressantes à examiner en ce qu'elles montrent comment s'entrelacent le commun partagé et le singulier.

\section{L'intervention de Farouk}

Comme nous l'avons mentionné plus haut, outre sa prise de parole qui modifie l'orientation du tour de table, Farouk interrompt son déroulement à deux reprises pour parler de contenus liés au thème de la musique, thème de la prochaine émission. Chacune de ses tentatives intervient à un point de transition, c'est-à-dire au moment où le transfert de la parole entre la personne qui est en train de parler et la suivante va s'effectuer. Il procède en levant le doigt et en regardant l'animatrice, afin de lui demander la parole.

Observons le moment où Farouk intervient à la fin du tour de Quentin, afin d'évoquer ses souhaits de musique pour l'émission. Sa tentative a lieu (1. 4) alors que l'animatrice recadre justement l'intervention de Quentin (1. 2) qui est en train d'ouvrir potentiellement une discussion autour de l'interview de son frère :

\section{Extrait 13 : Intervention de Farouk au cours du tour de table}
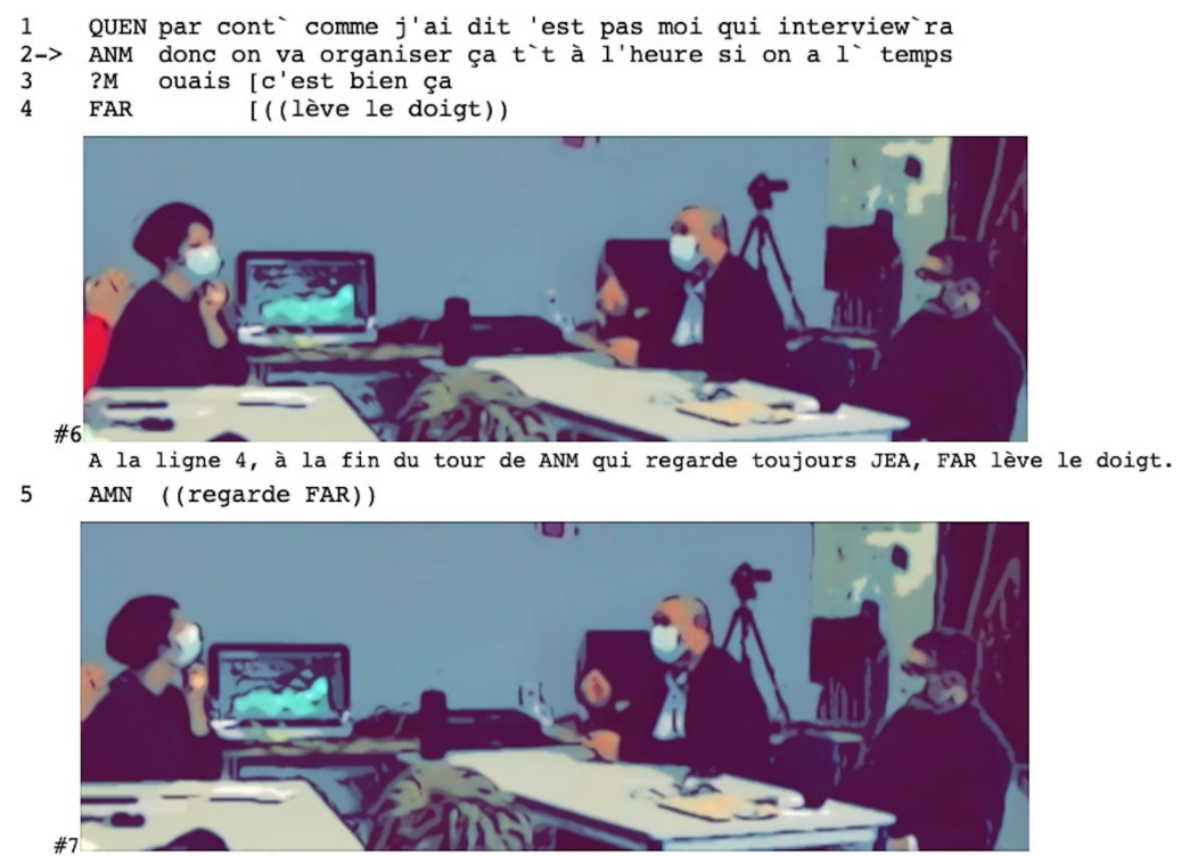

AMN attribue la parole à FAR par un regard et un mouvement de tête.

ARM (ça s'ra)

FAR moi j'aime (.) si c'est le thème euh: : sur la musique j'aimerais qu'on arrive à si c'est possible de visionner un peu la musique orientale 
Le développement de Farouk dure une dizaine de lignes, pendant lesquelles l'animatrice prend note sur son cahier et formule son accord verbalement et en opinant de la tête. Puis elle reprend la parole et relance le cours du tour de table (lignes 22, 24) :

\section{Extrait 14 : Intervention de Farouk au cours du tour de table}

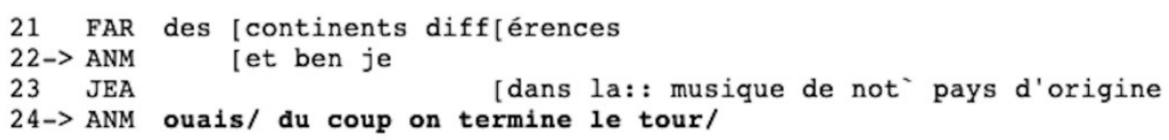

Ce recadrage de l'activité arrive au moment où, d'une part, il est question de différences ( « on est un peu différentiels euh des personnes », " des continents différences »), et, d'autre part, où la proposition de Farouk risque de devenir un sujet de discussion dans le groupe, puisque JEA enchaîne, ligne 23. L'animatrice interrompt l'embryon de discussion, en suggérant aux personnes suivantes dans le tour de table d'exprimer, si elles le veulent, leurs souhaits ou idées pour l'atelier quand elles parleront :

\section{Extrait 15 : Clôture du tour de Farouk par l'animatrice}

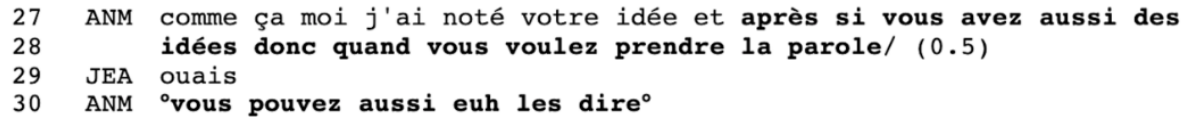

L'intervention de Farouk est intéressante dans la discussion autour de « faire le pair », parce qu'elle se distingue : elle intervient à un moment non attendu du tour de table, elle relève d'une activité différente de celle qui est en cours, et elle évoque des différences (« comme vous avez dit on est un peu différentiels euh des personnes »), sans qu'il soit très clair s'il s'agit de différences entre les personnes du groupe (chacun sa musique), ce qui peut conduire au partage, comme le comprend JEA (ligne 23) ou de différences du groupe par rapport à l'extérieur.

\section{L'intervention de Catherine}

La prise de parole de Catherine se distingue aussi de celle des autres, même si elle semble au départ bien ajustée au cadre. Catherine se présente en effet à la fois en écho aux demandes de ANM (prénom, investissement dans le groupe Radio et intérêt pour la musique), et par rapport à la prise de parole précédente, celle de Valentine (extrait 12), à qui elle reprend le mot « découverte » (« pour moi c'est une découverte aussi depuis::: (0.3) y a quinze jours en arrière »). Elle appuie ses propos sur son amour de la musique par l'énumération des instruments qu'elle a appris, ce qui la conduit à raconter avoir abandonné la guitare commencée au GEM avec Orel :

\section{Extrait 16 : Catherine}

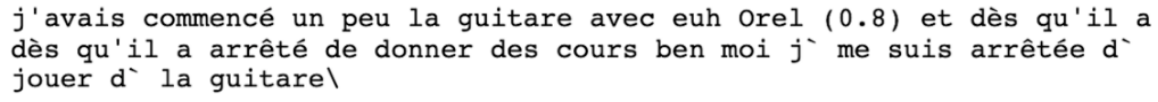

Cette évocation dure assez longtemps et se fait sur une tonalité sonnant comme une plainte ( « mon père et mon entourage ont trouvé ça dommage mais moi seule j'y arrivais pas », « on m` disait mais (0.4) suis un tuto sur euh internet euh: (0.5) déjà j'avais pas la place pour tenir euh: (0.4) entre le: l'ordi et puis ma guitare et: .hh (0.4) tout ça », " la guitare est toujours chez moi mais: (.) elle joue plus », ces différents énoncés étant séparés de longues pauses). La durée et la tonalité de ce récit font qu'il n'est pas traité par les autres comme une information que l'on écoute silencieusement (comme dans un tour de table), mais comme un sujet ouvert qui attend des réactions. Ceci conduit à un long développement (une page dans la transcription), où chacun y va de son conseil (« prend un livre », « va à la MJC ») ou, pour 
l'animatrice, des commentaires et interprétations, et qui provoque un bref moment de désordre où tout le monde parle plus ou moins en même temps.

Comme Farouk, mais par d'autres moyens que lui, Catherine fait dévier l'activité en cours. Dans la séquence initiée par son récit, les participants ne sont plus en train de faire tous la même chose successivement (se présenter), mais se penchent sur son histoire.

\section{Conclusion}

La notion d'entraide mutuelle «ne détermine pas seulement des personnes "semblables", mais un mode d'échange qui n'est pas de l'ordre d'une prise en charge et repose sur la reconnaissance d'un "en commun" et d'une appartenance à un collectif. » (Launat, $2020: 42$ ). Si l'on suit cette idée, ce n'est peut-être pas le fait d'avoir vécu une expérience commune des troubles psychiques ou de l'isolement qui fait des adhérents des pairs. Au contraire, ce serait le fait même d'être adhérents au GEM qui créerait de la parité. Les adhérents acquièrent progressivement cette expérience commune associative, expérimentent le GEM et ses usages et appartiennent progressivement à la communauté des gemeurs.

Le GEM est un espace au sein duquel les adhérents peuvent se redéfinir. Il leur permet de mettre en avant d'autres aspects identitaires, en dehors du fait d'être une personne concernée par des troubles psychiques ou en situation d'isolement. Dans cet espace singulier, la maladie est mise entre parenthèses (Troisoeufs, 2009). Le GEM permet de vivre par-delà le trouble. Il laisse donc la possibilité aux adhérents de créer d'autres formes de parité. Ils peuvent être concernés (ou non) par les troubles psychiques, être en situation de handicap, vivre l'isolement social au quotidien, mais ce n'est pas nécessairement cet aspect identitaire qui va créer de la proximité avec un autre adhérent. L'atelier Radio est un exemple parmi d'autres du fait qu'un adhérent peut se sentir proche d'un autre, le considérer subjectivement comme un pair parce qu'ils partagent tous deux un même intérêt ou une même passion pour une pratique et participent chaque semaine au même atelier. Ce pourrait être parce qu'ils vivent dans le même quartier ou le même foyer. Ils partagent des savoirs issus d'expériences de vie ordinaire (Gardien, 2020). L'analyse d'un extrait de l'atelier donne une première idée de comment le sentiment de cette expérience commune du monde se construit dans la réalité des pratiques. On voit bien à travers les échanges que, non seulement être pair ne se décrète pas, mais que cela relève véritablement d'un faire (on pourrait presque parler d'un travail), et que la parité, loin d'être la mêmeté, inclut toute une gamme de variétés et différences.

\section{Références}

Becker Howard. (1985). Outsiders: Études de sociologie de la déviance (chapitre Comment on devient fumeur de marijuana, p. 64-82). Paris : Éditions Métailié.

Blondiaux Loïc. (2008). Le Nouvel Esprit de la démocratie. Actualité de la démocratie participative. Paris : Seuil.

Chambon Nicolas, Traverso Véronique, Zeroug-Vial Halima. (2020). Médiateurs, pairs, interprètes... au secours du soin. Rhizome, vol. 75-76, p. 7-16.

Ebersold Serge. (2002). Le champ du handicap, ses enjeux et ses mutations : du désavantage à la participation sociale. Handicap - Revue des sciences humaines et sociales, vol. 94-95, p. 149-164.

Ehrenberg Alain. (2020). L'idéal du potentiel caché. Le rétablissement, le rite et la socialisation du mal. Anthropologie\&Santé, vol. 20, http://journals.openedition.org/ anthropologiesante $/ 6059$, consulté le 28 octobre 2021 
Ehrenberg Alain. (2021). Les changements de l'esprit du soin : le potentiel, le handicap et la forme de vie. Cliniques, vol. 21, p. 24-39.

Fiacre Patricia, Perrot-Dessaux Charlotte, Demoustier Séverine, Prieto Jacques, Raharijaona Mamy. (2017). Entre vulnérabilité et autonomie. Etude sur les groupes d'entraide mutuelle de la région Centre-Val de Loire. CREAI du Centre-Val de Loire, décembre 2017.

Gardien Eve. (2018). L'accompagnement et le soutien par les pairs. Grenoble : PUG.

Gardien Eve. (2020). Pairjectivité : des savoirs expérientiels ni objectifs, ni subjectifs. Éducation et socialisation, vol. 57, http://journals.openedition.org/edso/12581, consulté le 4 novembre 2021.

Gardien Eve, Laval Christian. (2018). The institutionalisation of peer support in France: development of a social role and roll out of public policies. ALTER European Journal of Disability Research, vol. 13, p. 69-82.

Goffman Erving. (1974). Les rites d'interaction. Paris : Éditions de Minuit.

Golf Graziella, d'Hauteville Thomas, Molinié Magali. (2020). "Faciliter» un groupe d'entraide du REV France, l'expérience de Montpellier. Rhizome, vol. 75-76, p. 193202.

Greco Luca. (2006). La présentation de soi dans un tour de table : identité, contexte et pratiques sociales. Verbum, vol. XXVIII, n²-3, p. 153-174.

Heijboer Claire. (2019). L'expertise usagère : un défi pour les institutions sociales et médicosociales du 21ème siècle. L'Usagent et le Maïticien. Thèse de Doctorat en Sciences de l'éducation. Paris : Université de Paris 5.

Joseph Isaac. (1998). Erving Goffman et la microsociologie. Paris : PUF.

Launat Dominique. (2020). De qui suis-je le pair? Pratiques en santé mentale, vol. 66, p. 40-49.

Laval Christian, Gardien Eve. (2020). Accompagner ses pairs n'est pas une activité de médiation, pourtant ils sont appelés « médiateurs de santé-pairs ».... Rhizome, vol. 75-76, p. 194-192.

Le Callonnec Aziliz. (à paraître, 2021). De quoi l'inclusion est-elle le nom ? Pratiques et postures professionnelles des animateurs de groupes d'entraide mutuelle. Rhizome, vol. 83.

Molinier Pascale. (2010). Au-delà de la féminité et du maternel, le travail du care. Champ psy, vol. 58, p. 161-174.

Niard Camille, Maugiron Philippe, Franck Nicolas. (2020). Le pair-aidant professionnel : un nouvel acteur pour de nouvelles réponses. Rhizome, vol. 75-76, p. 17-26.

Plantin Christian. (1993). Lieux communs, topoï, stéréotypes, clichés. Paris : Kimé.

Peintre Carole, Bibouda Mathilde, Bossu Marielle, Dusart Anne, Le Duff Rachelle, Marmont Thibault, Valiani Virginie, Tursi Pauline, Barreyre Jean-Yves et Ruiz Inca. (2017). Évaluation qualitative des effets produits par les GEM sur les situations de vie de leurs adhérents. ANCREAI, novembre 2017.

Sen Amartya. (2012). Repenser l'inégalité. Paris : Seuil.

Simon Pierre-Jean. (1991). Simmel : l'interaction sociale. In P.-J. Simon (éd.), Histoire de la sociologie. Paris : PUF, p. 435-450.

Traverso Véronique. (1993). Les routines : lieux communs de la conversation. In C. Plantin (éd.). Lieux communs, topoï, stéréotypes, clichés. Paris : Kimé, p. 111-123. 
Traverso Véronique. (1997). La pluie et le beau temps dans les conversations quotidiennes : aspects rituels et thématiques. Sciences de la société, vol. 41, p. 145-165.

Traverso Véronique. (2012). Longues séquences dans l'interaction : ordre de l'activité, cadres participatifs et temporalités. Langue française, vol. 175, p. 53-73.

Traverso, Véronique. (2021). Fragments de la vie d'avant : une forme de récit dans des séances de soutien psychosocial pour des femmes réfugiées. Langage et société, vol. 173, p. 83-113.

Troisoeufs Aurélien. (2009). La personne intermédiaire. Hôpital psychiatrique et groupe d'entraide mutuelle. Terrain, vol. 52, n¹, p. 96-111.

Troisoeufs Aurélien. (2012). Le passage en actes : du malade mental à la personne liminaire. Anthropologie des associations d'usagers de la psychiatrie. Thèse de Doctorat en Anthropologie. Paris : Université Paris Descartes.

Troisoeufs Aurélien. (2020). Patients intervenants, médiateurs de santé-pairs : quelles figures de la pair-aidance en santé ? Rhizome, vol. 75-76, p. 27-36.

Troisoeufs Aurélien, Eyraud Benoit. (2015). Psychiatrisés en lutte, usagers, Gemeurs,... : une cartographie des différentes formes de participation. Rhizome, vol. 58, p. 3-4.

Zask Joëlle. (2011). Participer. Essai sur les formes démocratiques de la participation. Paris : Le Bord de l'eau. 